\title{
THE EFFECT OF UNBALANCE MASS ON THE NECESSARY CONDITIONS OF THE DOUBLE-SUPPORT ROTOR AUTOBALANCING STABILITY
}
A.N. Gorbenko ${ }^{1}$
gan0941@yandex.ru
S.Kh. Shmelev ${ }^{2}$
shm_Novo@mail.ru
G. Strautmanis ${ }^{3}$
guntis.strautmanis@rtu.lv

${ }^{1}$ Kerch State Maritime Technological University, Kerch, Republic of Crimea, Russian Federation

${ }^{2}$ Admiral Ushakov State Maritime University, Novorossiysk, Russian Federation

${ }^{3}$ Riga Technical University, Riga, Latvia

\begin{abstract}
The analysis of necessary conditions for autobalancing stability of rotor, which performs spatial oscillations, was carried out in this paper taking into account the influence of unbalance and autobalancer masses. It was found that the using of traditional models, where unbalance and autobalancer masses are assumed small, could lead to significant errors in the dynamics analysis of spatially moving rotor. The influence of this factor leads to the doubling of the critical rotational speeds spectrum. Moreover, the system motion between the split critical frequencies is unstable. There may be one or two onset areas of autobalancing mode motion depending on the dynamic rotor type, its location relative to the supports, the unbalance mass and other system parameters. It was found that rotors, that are long type or close to the spherical type, are the most sensitive to this factor. On the other hand, rotors of the short type are weakly sensitive. It is shown that the most preferred case is when the unbalance and autobalancer location plane passes through the common mass center of the composite rotor. The quantitative criterion is formulated for the necessity of taking into account (or not taking into account) the influence of this factor when analyzing the system dynamics
\end{abstract}

\section{Keywords}

Rotor, unbalance, autobalancing stability, critical speeds, anisotropy

Introduction. The unbalance of the rotor is the main reason for the increased vibration of rotor type machines. This factor leads to a decrease in the functional properties, reliability and resource of rotary mechanisms. To 
A.N. Gorbenko, S.Kh. Shmelev, G. Strautmanis

overcome this problem, passive-type autobalancing devices can be successfully used, which are able to compensate automatically the current rotor unbalance. However, the actual use of autobalancing devices is faced with the problem of their work stability.

Autobalancing devices have been known for a long time. Studies of the rotor dynamics with autobalancers can be found in [1-7], etc. In the monograph [2], a very comprehensive review of works on the theory of autobalancing is given. Autobalancing of a rotor that performs spatial motion was studied in $[2,4,5,7]$ and others.

In the well-known works, it is traditionally assumed that the unbalance mass of the rotor and compensating autobalance weights is substantially less than the mass of the rotor itself. This approach provides a certain simplification of the system mathematical model and its study. However, the analysis carried out in $[8,9]$ showed that the rotor dynamics from the autobalancing device, which performs spatial movement, has more complex properties, which depend on the mass of the unbalance and compensating autobalance weights. The qualitative difference is that a real unbalanced rotor with an autobalancing device exhibits an inertial anisotropy property, which results in splitting (doubling) of its critical rotational frequencies [10]. In the same paper, a criterion was formulated for the necessity of taking into account (or not taking into account) the influence of this factor when analyzing the system dynamics.

In [11], the necessary conditions for the stability of the rotor autobalancing were first obtained, taking into account the influence of the unbalance magnitude and the mass of the autobalancer. Analytical research has shown that the influence of this factor is non-trivial.

The purpose of this work is to analyze the influence of the unbalance mass and other system parameters on the necessary conditions for the autobalance stability of a double-support rotor that performs a spatial motion. Besides, the rotor is arbitrarily located relative to the supports. This paper is a continuation of the work [11].

Physical model and mechanical system parameters. The mechanical system "rotor-multi-mass autobalancer" is considered (Fig. 1). A rigid rotor with a mass $M_{r}$ based on two elastic-viscous isotropic supports and rotates with an angular velocity $\omega$. The rotor center of mass is located at point $\mathrm{G}$. Polar $\mathrm{Cr}$ and equatorial $\mathrm{Ar}$ moments of inertia about its own axes characterize the inertial properties of the rotor in its spatial movement. The rotor disk in the general case can be based between both the supports and have a cantilever arrangement.

The unbalance of the rotor is modeled by the presence of a point unbalanced mass $m_{0}$ rigidly attached to the rotor at a distance of $R_{0}$ from the axis of rotation 


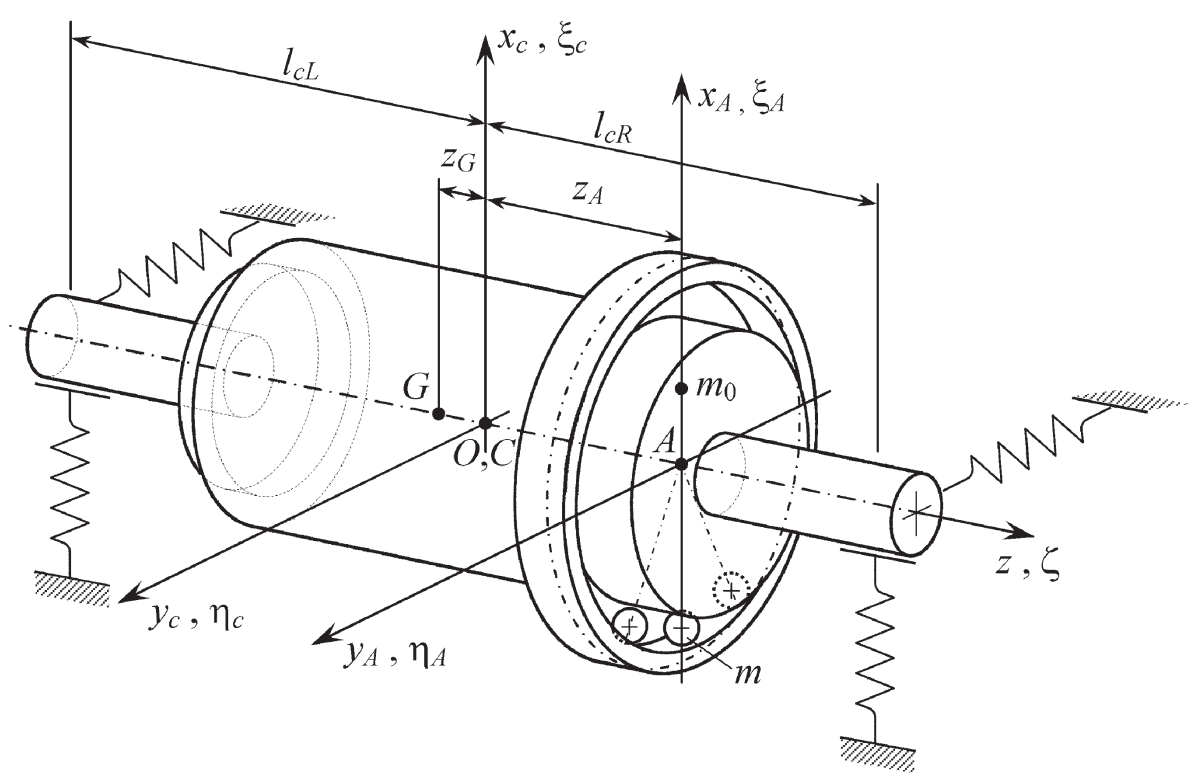

Fig. 1. The unbalanced double-support rotor with autobalancer

in the plane $A$. In the transverse plane $z=z_{A}$, an autobalance is attached to the rotor with a set of compensating solid-state weights (balls, rollers, etc.). The common center of mass of the composite rotor with an unbalanced mass and an autobalancer is located at point $C$ on the axis of rotor rotation.

In the considered physical model, it is assumed that the unbalanced mass and the compensating weights masses of the autobalancing device are considerable and can be comparable with the mass of the rotor. As a result, the composite rotor acquires qualitatively new dynamic properties. This property distinguishes this model from the existing ones. The rotor motion is described in the fixed coordinate system $x y z$ and in the rotating axes $\xi \eta \zeta$. The origin of $O$ coincides with the common center of mass of the system (point $C$ ) with a stationary rotor.

A complete description of the physical model parameters of the "rotormulti-mass autobalancer" mechanical system and the derivation of its motion system of equations are given in $[9,10]$.

We note that to obtain the necessary conditions for the autobalancing stability using the engineering phase criterion [11], it is sufficient to consider a simpler physical model. In this case, the motion of an unbalanced rotor with an unbalanced mass, but without an autobalancer, is investigated.

The mechanical system under consideration is characterized by the following main dimensionless parameters: 
$\Omega=\omega / p_{x}$ is the dimensionless rotor speed;

$\bar{C}_{\Sigma 0}=C_{\Sigma 0} / A_{\Sigma 0}$ is the dimensionless parameter characterizing the type of rotor: the short type $\left(\bar{C}_{\Sigma 0}>1\right)$, the long type $\left(\bar{C}_{\Sigma 0}<1\right)$ or the spherical type $\left(\bar{C}_{\Sigma 0}=1\right)$

$\bar{m}_{0}=m_{0} / M_{s}$ is the dimensionless unbalance mass characterizing static and dynamic unbalance of the rotor;

$\Delta \bar{A}_{0}$ is the dimensionless parameter characterizing the magnitude of the inertial anisotropy of the unbalanced rotor, caused by the influence of the unbalanced mass of the rotor;

$\bar{p}_{x}=1, \bar{p}_{\alpha}, \bar{c}_{y \alpha}$ are dimensionless parameters depending on the respective masses, moments of inertia and stiffness of the supports, which characterize the influence of the rotor installation pattern relative to the supports (intersupport symmetric, inter-support non-symmetric, cantilever);

$\bar{z}_{A 0}$ is the dimensionless distance of the unbalance mass and autobalancer from the common center of mass of the rotor system.

For a complete description of dimensional and dimensionless parameters of a mechanical system, see [9-11].

Necessary conditions for the autobalancing stability. The following necessary stability conditions for autobalancing a double-support rotor were obtained in [11]:

$$
\frac{\Delta \bar{I}_{02}+\bar{z}_{A 0}^{2}}{\Delta \bar{I}_{02}} \frac{\Omega^{2}-\Omega_{a}^{2}}{\left(\Omega^{2}-\Omega_{K 2}^{2}\right)\left(\Omega^{2}-\Omega_{K 4}^{2}\right)}>0,
$$

where

$$
\Delta \bar{I}_{02}=1-\bar{C}_{\Sigma 0}-\Delta \bar{A}_{0} ; \Omega_{a}^{2}=\frac{\bar{p}_{\alpha}^{2}+2 \bar{z}_{A 0} \bar{c}_{y \alpha}+\bar{z}_{A 0}^{2}}{\Delta \bar{I}_{02}+\bar{z}_{A 0}^{2}} ;
$$

$\Omega_{K 2,4}$ are upper values of the split critical rotor speeds; $\Omega_{a}$ is the antiresonance critical rotor speed.

Conditions (1) are obtained using the engineering phase criterion for the autobalancing occurrence. Physically, these conditions show that the autobalancing mode of movement can take place only under the condition of antiphase movement of the rotor axis balanced point (point $A$ ) with respect to the direction of the unbalance caused by the rotor unbalance mass.

The critical speeds of a spatially moving rotor, included in conditions (1), are determined by expressions for the general case of system parameters [10]: 


$$
\begin{aligned}
& \Omega_{K 1,2}^{2}=\frac{1}{2}+\frac{\bar{p}_{\alpha}^{2}}{2 \Delta \bar{I}_{01,2}}-\frac{1}{2} \sqrt{\left(1-\frac{\bar{p}_{\alpha}^{2}}{\Delta \bar{I}_{01,2}}\right)^{2}+4 \frac{\bar{c}_{y \alpha}^{2}}{\Delta \bar{I}_{01,2}}} ; \\
& \Omega_{K 3,4}^{2}=\frac{1}{2}+\frac{\bar{p}_{\alpha}^{2}}{2 \Delta \bar{I}_{01,2}}+\frac{1}{2} \sqrt{\left(1-\frac{\bar{p}_{\alpha}^{2}}{\Delta \bar{I}_{01,2}}\right)^{2}+4 \frac{\bar{c}_{y \alpha}^{2}}{\Delta \bar{I}_{01,2}}},
\end{aligned}
$$

where $\Delta \bar{I}_{01,2}=1-\bar{C}_{\Sigma 0} \pm \Delta \bar{A}_{0}$.

The considered rotor can have from one to four critical rotational speeds $\Omega_{K i}, i=1,2,3,4$, depending on the values of the system parameters. The values $\Omega_{K 1,2}=\Omega_{K x 1,2}$ correspond to the transverse oscillations of the rotor, and $\Omega_{K 3,4}=\Omega_{K \alpha 1,2}$ correspond to the angular oscillations of the rotor with typical values of the system parameters. Moreover, in the ranges between the split critical velocities (i.e., for at $\Omega_{K 1}<\Omega<\Omega_{K 2}$ and $\Omega_{K 3}<\Omega<\Omega_{K 4}$ ), the rotor movement is unstable due to its inertial anisotropy [12, etc.].

Thus, autobalancing of the rotor is generally possible in two ranges of rotational speed:

$$
\Omega_{1}<\Omega<\Omega_{2} \text { and } \Omega>\Omega_{3},
$$

where

$$
\begin{gathered}
\Omega_{1}=\min \left(\Omega_{K 2}, \Omega_{a}, \Omega_{K 4}\right) ; \Omega_{2}=\min \left(\Omega_{a}, \Omega_{K 3}\right) ; \\
\Omega_{3}=\max \left(\Omega_{K 2}, \Omega_{a}, \Omega_{K 4}\right) ; \Omega_{1}<\Omega_{2}<\Omega_{3} .
\end{gathered}
$$

Here, for formalization, we used a set of critical (boundary) rotational speeds $\Omega_{1,2,3}$, which is ordered in ascending order from $\Omega_{K 2}, \Omega_{K 3}, \Omega_{K 4}, \Omega_{a}$ values, see (1), (2). For traditional rotary machines, there are relations: $\Omega_{K 2}<\Omega_{a}<\Omega_{K 3}<\Omega_{K 4}$, i.e., $\Omega_{1}=\Omega_{K 2}, \Omega_{2}=\Omega_{a}, \Omega_{3}=\Omega_{K 4}$. However, depending on the parameters of the system, the intermediate boundary velocity $\Omega_{2}$ can be equal to the value of both $\Omega_{a}$ and $\Omega_{K 3}$, which takes into account the possibility of a loss of stability of oscillations of an inertia-anisotropic rotor.

The necessary conditions for the stability of autobalancing (1) summarize the known conditions obtained for a spatially moving rotor in [2], since they take into account the peculiar influence of an unbalanced mass on the dynamic properties of a rotating rotor.

For clarity, the Fig. 2 shows the onset areas location of the rotor autobalance, taking into account the influence of the unbalance and areas of stability loss.

The influence analysis of system parameters on rotor autobalancing stability conditions. The necessary stability conditions for the autobalancing 
A.N. Gorbenko, S.Kh. Shmelev, G. Strautmanis

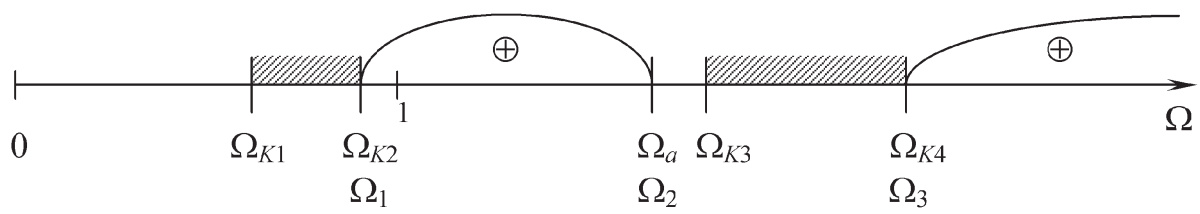

Fig. 2. Areas of rotor speed $\Omega$, within which its autobalancing is possible, taking into account the influence of unbalance mass:

$\oplus$ are areas of the anti-phase rotor movement; $\mathscr{W}$ is the motion instability of inertial-anisotropic rotor

of a spatially moving rotor (3) depend in a nontrivial way on the system parameters. A qualitative analysis of this influence was performed in [11]. Below are the results of numerical analysis.

In Fig. 3-5 shows graphs characterizing the areas of system parameters in which rotor autobalancing is possible. The graphs are plotted with the following basic values of the parameters: $\bar{C}_{\Sigma 0}=0.75 ; \bar{\rho}_{s}=0.4 ; \Delta \bar{A}_{0}=0.1 ; \bar{z}_{A 0}=0.8$; $\bar{m}_{0}=0.05 ; \bar{R}_{0}=2 ; \bar{A}_{m 0}=0.2$. In addition, in the case of an asymmetrically installed rotor $\left(\bar{l}_{L}=0.8\right)$, we have $\bar{p}_{\alpha}=1.46 ; \bar{c}_{y \alpha}=0.75 ; \Omega_{K 1-4}=(0.837 ; 0.849$; $2.52 ; 3.80) ; \Omega_{a}=2.24$; for a symmetrically mounted rotor $\left(\bar{l}_{L}=0.5\right)$, we have $\Omega_{K 1-4}=\bar{p}_{\alpha}=1.25 ; \bar{c}_{y \alpha}=0 ; \Omega_{K 1-4}=(1 ; 1 ; 2.11 ; 3.23) ; \Omega_{a}=1.67$ and for the cantilever rotor $\left(\bar{l}_{L}=1.2\right)$ we have $\bar{p}_{\alpha}=2.15, \bar{c}_{y \alpha}=1.75, \Omega_{K 1-4}=(0.567$; $0.575 ; 3.73 ; 5.61), \Omega_{a}=3.20$. Calculations were carried out using formulas (1)-(3), see also works [9-11].

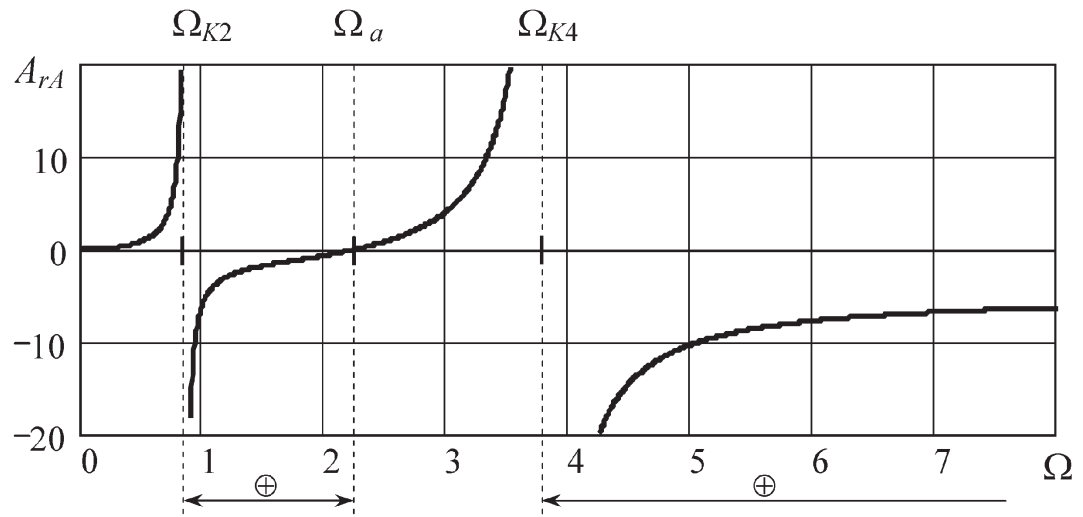

Fig. 3. Amplitude-frequency characteristic of the unbalanced rotor point $A$ motion (taking into account the sign of the point deviation):

$\oplus$ is the anti-phase rotor movement, where autobalance is possible

The amplitude-frequency characteristic of rotor oscillations in Fig. 3 illustrates the arrangement of the rotational frequency ranges of the unbalanced 


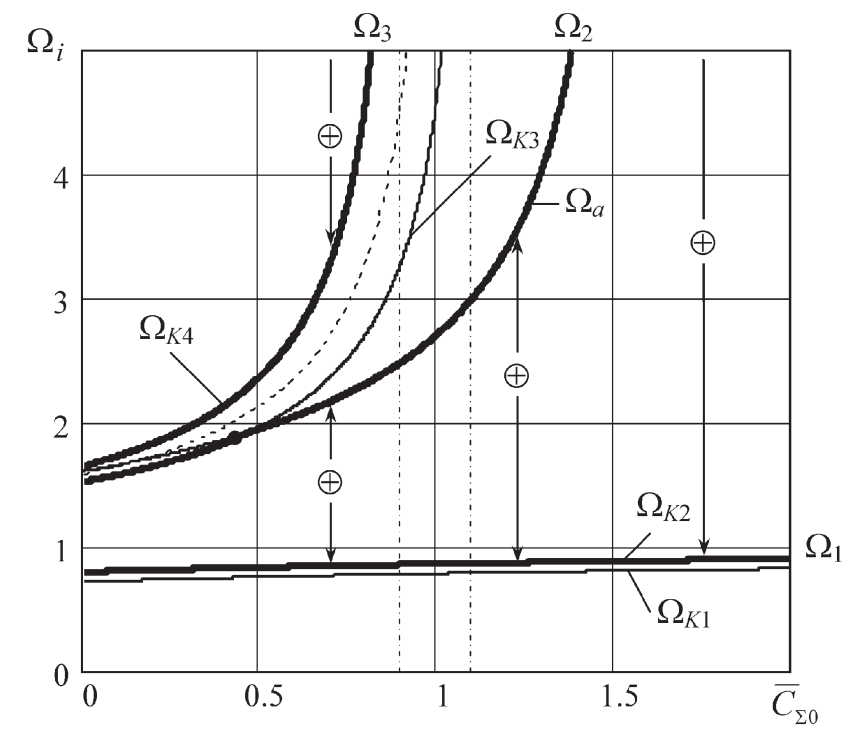

Fig. 4. The necessary boundaries $\Omega_{1,2,3}$ of the rotor autobalancing depending on the rotor type $\bar{C}_{\Sigma 0}$ :

is the $\Omega_{K 3,4}$ for isotropic rotor; $\Delta \bar{A}_{0}=0$; $\oplus$ is the anti-phase rotor movement, where autobalance is possible
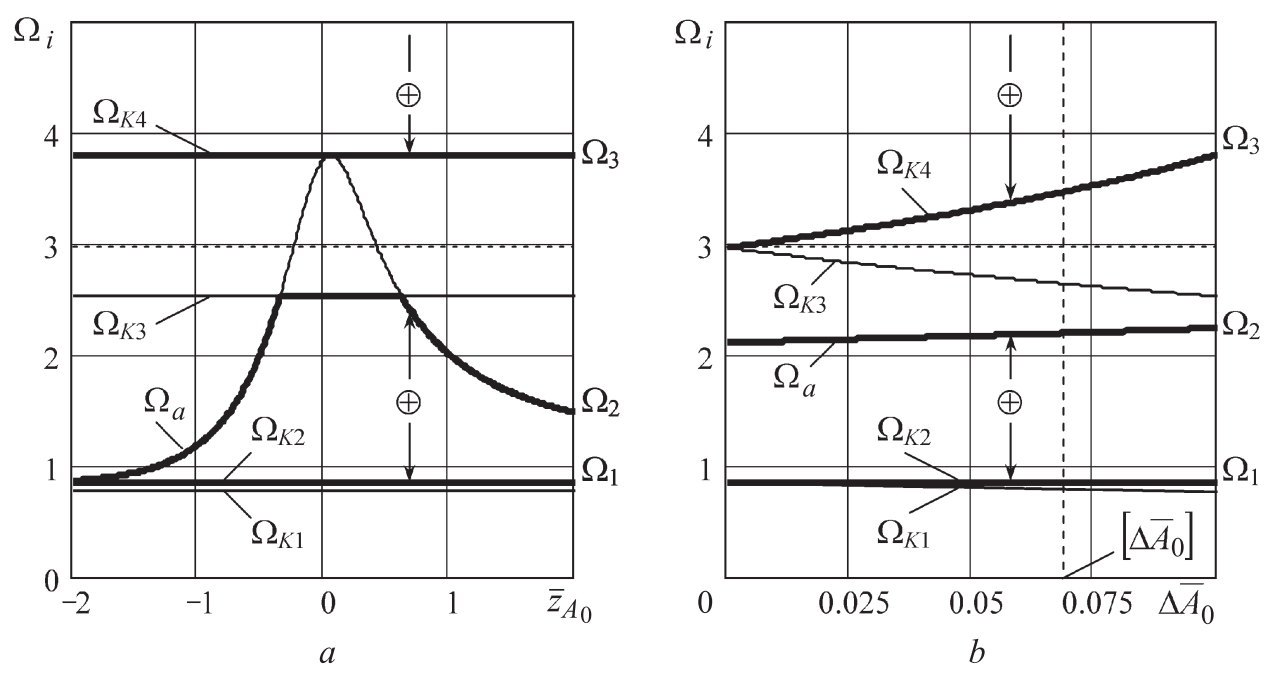

Fig. 5. The necessary boundaries $\Omega_{1,2,3}$ of the rotor autobalancing for asymmetrically located long rotor $\left(\bar{l}_{L}=0.8\right)$ depending on:

$a$ is the unbalanced mass location $\bar{z}_{A 0} ; b$ is the degree of the rotor inertial anisotropy $\Delta \bar{A}_{0}$, caused by the influence of unbalance mass

rotor, within which the anti-phase movement of the balanced point $A$ on its axis takes place. In this case, the rotor is of a long type and asymmetrically mounted with respect to the supports. In the ranges of $\Omega$, where the amplitude of the 
lateral displacement of the rotor is negative: $A_{r A}=\left|\vec{r}_{A}\right|=\bar{\xi}_{A}<0$, the rotor deviates from the axis of rotation in the direction opposite to the direction of the unbalance force action $\vec{S}_{0}$. In these ranges, the onset of the autobalancing mode of the rotor system is possible.

In Fig. 4 shows the rotor type (parameter $\bar{C}_{\Sigma 0}$ ) affecting on the number and location of areas in which autobalancing is possible. The calculations are performed on the example of an asymmetrically located rotor $\left(\bar{l}_{L}=0.8\right)$. The graphs illustrate the features of autobalancing rotors of the long, spherical and short types formulated in [11]. It follows from the graphs that:

a) a long rotor $\left(\bar{C}_{\Sigma 0}<\left(1-\Delta \bar{A}_{0}\right)\right)$ can be autobalanced in two ranges of rotational speeds: at $\Omega_{1}<\Omega<\Omega_{2}$ and at $\Omega>\Omega_{3}$;

b) a rotor close to spherical type $\left(1-\Delta \bar{A}_{0}\right)<\bar{C}_{\Sigma 0}<\left(1-\Delta \bar{A}_{0}+\bar{z}_{A 0}^{2}\right)$, allows autobalancing in one rotor speed range, bounded above and below when $\Omega_{1}<\Omega<\Omega_{2}$;

c) a short rotor $\left(\bar{C}_{\Sigma 0}>\left(1-\Delta \bar{A}_{0}+\bar{z}_{A 0}^{2}\right)\right)$ has one range of rotational speed, bounded below when $\Omega>\Omega_{1}$.

Graphs in Fig. $5 a$ show, that the location of the unbalance and autobalance has a noticeable effect on the ability to autobalance. From this point of view, the most favorable case is when the plane of location of the unbalance mass and the autobalancing device passes through a common center of mass $C$ of the composite rotor, i.e., parameter $\bar{z}_{A 0}=0$. Increasing the absolute value of the parameter $\left|\bar{z}_{A 0}\right|>0$ leads to a narrowing of the areas of possible rotor autobalancing.

In Fig. $5 b$ using the example of a long rotor, the effect of the inertial anisotropy $\Delta \bar{A}_{0}$ of the composite rotor (which, in turn, is caused by the influence of the unbalanced mass $\bar{m}_{0}$ ) on the possibility of autobalancing is shown. Recall that it is the consideration of this factor that distinguishes this work from the existing results. It follows from the graphs that an increase in $\Delta \bar{A}_{0}$ leads to a slight expansion of the "lower" area of possible autobalancing, where $\Omega_{1}<\Omega<\Omega_{2}$. However, the "upper" area of possible autobalancing is noticeably narrowing, where $\Omega>\Omega_{3}$. In general, the unbalanced mass $m_{0}$ and the inertial anisotropy $\Delta A_{0}$ caused by the mass lead to a deterioration in the possibility of autobalancing the rotor.

Because of the analysis, it was found that the mass of the unbalance and the autobalance can significantly affect the values of the critical rotational speeds (primarily in terms of angular oscillations), which are the boundaries of

78 ISSN 0236-3941. Вестник МГТУ им. Н.Э. Баумана. Сер. Машиностроение. 2019. № 2 
the areas where the rotor is automatically balanced (see Fig. 5b). Therefore, the actual question is, in which cases it is necessary to take into account the mass of unbalance and autobalance in the theoretical analysis of the system. In [10], the following formal criterion was proposed for the need to take into account the influence of unbalance mass and autobalance

$$
\delta \Omega_{K \alpha}=\max \left(\left|\delta \Omega_{K \alpha 1,2}\right|\right)>\left[\delta \Omega_{K \alpha}\right],
$$

where $\delta \Omega_{K \alpha 1,2}=\frac{\Omega_{K \alpha 1,2}-\Omega_{K \alpha 0}}{\Omega_{K \alpha 1,2}}=1-\sqrt{1 \pm \frac{\Delta \bar{A}}{1-\bar{C}_{\Sigma}}} ; \delta \Omega_{K \alpha}$ is the coefficient of the rotor sensitivity to inertial anisotropy, due to the unbalanced mass and autobalancer. This coefficient represents the largest relative change in the critical speeds of the angular oscillations of the rotor; $\left[\delta \Omega_{K \alpha}\right]$ is the permissible error of determining the values of the critical speeds of the angular oscillations of the rotor. It is recommended to take the permissible value equal to $\left[\delta \Omega_{K \alpha}\right]=0.15$.

According to the physical meaning, criterion (4) reflects the requirement that the error in determining the critical rotational speeds does not exceed the normalized value. According to the expressions in (4), this criterion can also be expressed in terms of the maximum permissible value of the rotor inertial anisotropy $\left[\Delta \bar{A}_{0}\right]$, which is caused by the presence of an unbalance mass $\left[\bar{m}_{0}\right]$ :

$$
\Delta \bar{A}_{0}>\left[\Delta \bar{A}_{0}\right]=\left|1-\bar{C}_{\Sigma 0}\right|\left(1-\left(1-\left[\Delta \delta \Omega_{K \alpha}\right]\right)^{2}\right) .
$$

In the case of the criterion inequality (5) or (4) fulfillment, it is necessary to take into account the influence of the unbalanced mass and the autobalancing device mass on the mechanical system dynamics, i.e., to apply a refined model of the system movement and the necessary conditions for the autobalancing stability (1). For the numerical examples given earlier, the limit value of the unbalanced rotor inertial anisotropy is $\left[\Delta \bar{A}_{0}\right]=0.069$ (Fig. $5 b$ ), which corresponds to a relative unbalance mass $\left[\bar{m}_{0}\right]=0.017$. Otherwise, it is sufficient to use a simplified (traditional) model of the rotor system motion, in which the unbalance and the autobalancer masses are considered small values (of the first order of smallness).

Note that mentioned criterion (4), (5) is applicable to rotors of a long type and close to a spherical type. In these cases, the rotor has critical speeds of both angular $\Omega_{K_{\alpha 1,2}}$ and transverse $\Omega_{K x 1,2}$ vibrations. On the other hand, short-type rotors (when $\bar{C}_{\Sigma 0}>\left(1-\Delta \bar{A}_{0}+\bar{z}_{A 0}^{2}\right)$ ) are weakly sensitive to inertial anisotropy $\Delta \bar{A}_{0}$, since in this case the critical velocities of the angular oscillations are physically absent. For such rotors, it is possible to suppose that it is permissible to 
A.N. Gorbenko, S.Kh. Shmelev, G. Strautmanis

disregard the influence of the unbalanced mass and the autobalancing device on the dynamics of the mechanical system.

Conclusion. It is shown that the unbalance mass can significantly affect the dynamic properties of the mechanical system "unbalanced rotor-autobalancer". It has been established that the use of traditional models, where the unbalance and autobalancer masses are assumed small, can lead to significant errors in the analysis of the spatially moving rotor dynamics.

Analysis of the system parameters influence allowed us to establish the following.

1. Depending on the rotor dynamic type, there may be one or two onset areas of the autobalancing motion mode. Wherein:

- a long rotor can be automatically balanced in two ranges of rotational speed;

- a rotor close to a spherical type allows autobalancing in one speed range, bounded above and below;

- a short rotor has one range of rotational speed, bounded below.

2. The location of unbalance and autobalance has a noticeable effect on the autobalancing ability. From this point of view, the most favorable is the case when the plane of location of the unbalance mass and the autobalancing device passes through the common center of mass $C$ of the composite rotor. Increasing in one or the other direction of the distance between the center of mass $C$ and the plane of location of the unbalance mass and the autobalancing device lead to a narrowing of the areas of possible rotor autobalancing.

3. The possibility of the rotor autobalancing can significantly depend on the magnitude of the unbalance and autobalancer masses. Due to the influence of this factor, the rotor in more or less degree exhibits the property of inertial anisotropy, critical rotational speeds splitting (doubling) takes place and between these speeds, the motion of the system is unstable. The analysis showed that the most sensitive to this factor are long rotors and rotors, close to the spherical type.

4. With the help of the proposed criterion for the given numerical example, the limiting values of the rotor inertial anisotropy $\left[\Delta \bar{A}_{0}\right]$ (and the corresponding relative unbalance mass $\left[\bar{m}_{0}\right]$ ) are determined. If these limits are exceeded, the effect of unbalance and autobalancer masses on the stability of the rotor autobalance must be taken into account. Otherwise, as well as in the case of a short-type rotor, it suffices to use a simplified (traditional) model of the rotor system, in which the unbalance and autobalancer masses are considered small.

The results will be useful in the design and operation of rotor mechanisms with autobalancer.

Translated by V. Shumaev 
The Effect of Unbalance Mass on the Necessary Conditions...

\section{REFERENCES}

[1] Gusarov A.A. Avtobalansiruyushchie ustroystva pryamogo deystviya [Automatic balancing devices of direct action]. Moscow, Nauka Publ., 2002.

[2] Filimonikhin G.B. Zrivnovazhennya i vibrozakhist rotoriv avtobalansirami z tverdimi ko-riguvalnimi vantazhami [Balancing and vibration protection of rotors by autobalancers with solid corrective weights]. Kirovograd, KNTU Publ., 2004.

[3] Gorbenko A.N. On the stability of self-balancing of a rotor with the help of balls. Strength Mater., 2003, vol. 35, no. 3, pp. 305-312. DOI: 10.1023/A:1024621023821

[4] Rodrigues D.J., Champneys A.R., Friswell M.I., et al. Automatic two-plane balancing for rigid rotors. Int. J. Non-Linear Mech., 2008, vol. 43, no. 6, pp. 527-541.

DOI: 10.1016/j.jijonlinmec.2008.01.002

[5] Bykov V.G. Compensating of statically and dynamically unbalanced rotor by singleplane auto-balancing device. Vestnik Sankt-Peterburgskogo universiteta. Ser. 1. Matematika. Mekhanika. Astronomiya [Vestnik of Saint Petersburg University. Mathematics. Mechanics. Astronomy], 2009, no. 4, pp. 67-76 (in Russ.).

[6] Strautmanis G., Mezitis M., Strautmane V., et al. Impact of dimensions of the compensating mass of the automatic balancer on its acceleration. VP, 2017, vol. 12, pp. 1-5. DOI: 10.21595/vp.2017.18449

[7] Filimonikhin G., Filimonikhina I., Yakymenko M., et al. Application of the empirical criterion for the occurrence of auto-balancing for axisymmetric rotor on two isotropic elastic supports. Eastern-European J. Enterprise Technologies, 2017, vol. 2, no. 7, pp. 51-58. DOI: $10.15587 / 1729-4061.2017 .96622$

[8] Filimonikhin G.B., Gorbenko A.N. Effect of the balls mass of the autobalancer on structure of the motion equations of the rotor on two supports. Automation of production processes in mechanical and instrument engineering: Ukr. interdepart. sci.-tech. coll. $\mathrm{Na}$ tional University “Lviv Polytechnic", 2011, vol. 45, pp. 478-488 (in Russ.).

[9] Gorbenko A.N. Mass-inertial characteristics and dimensionless equations of twobearing rotor motion with auto-balancer in terms of compensating body mass. Nauka $i$ obrazovanie: nauchnoe izdanie [Science and Education: Scientific Publication], 2015, no. 12 (in Russ.). DOI: 10.7463/1215.0827773

[10] Gorbenko A.N. Auto-balancer influence on the critical speeds of rotor on two supports. Nauka i obrazovanie: nauchnoe izdanie [Science and Education: Scientific Publication], 2016, no. 10, pp. 143-167 (in Russ.). DOI: 10.7463/1016.0847756

[11] Gorbenko A.N., Shmelev S.Kh. Necessary self-balancing robustness conditions for a two-bearing rotor taking unbalance mass into account. Vestn. Mosk. Gos. Tekh. Univ. im. N.E. Baumana, Mashinostr. [Herald of the Bauman Moscow State Tech. Univ., Mechan. Eng.], 2018, no. 5, pp. 36-50 (in Russ.). DOI: 10.18698/0236-3941-2018-5-36-50

[12] Dimentberg F.M., Kolesnikov K.S., eds. Vibratsii v tekhnike. T. 3. Kolebaniya mashin, konstruktsiy i ikh elementov [Vibrations in the technique. Vol. 3. Oscillations of machines, constructions and their elements]. Moscow, Mashinostroenie Publ., 1980.

ISSN 0236-3941. Вестник МГТУ им. Н.Э. Баумана. Сер. Машиностроение. 2019. № 2 
A.N. Gorbenko, S.Kh. Shmelev, G. Strautmanis

Gorbenko A.N. - Cand. Sc. (Eng.), Assoc. Professor, Department of Marine Power Plants, Kerch State Maritime Technological University (Ordzhonikidze ul. 82, Kerch, Republic of Crimea, 298309 Russian Federation).

Shmelev S.Kh. - Cand. Sc. (Eng.), Assoc. Professor, Department of Marine Heat Engines, Admiral Ushakov Maritime State University (Lenina prospekt 93, Novorossiysk, 353918 Russian Federation).

Strautmanis G. - Dr. Sc. (Eng.), Assoc. Professor, Department of Railway Transport, Faculty of Mechanical Engineering, Transport and Aeronautics, Riga Technical University (Kalku street 1, Riga, LV-1658 Latvia).

\section{Please cite this article as:}

Gorbenko A.N., Shmelev S.Kh., Strautmanis G. The effect of unbalance mass on the necessary conditions of the double-support rotor autobalancing stability. Herald of the Bauman Moscow State Technical University, Series Mechanical Engineering, 2019, no. 2, pp. 71-82. DOI: 10.18698/0236-3933-2019-2-71-82

82 ISSN 0236-3941. Вестник МГТУ им. Н.Э. Баумана. Сер. Машиностроение. 2019. № 2 